\title{
THE VALUE-SEMIGROUP OF A ONE-DIMENSIONAL GORENSTEIN RING
}

\author{
ERNST KUNZ
}

In a conversation about [4], O. Zariski indicated to the author that there should be a relation between Gorenstein rings and symmetric value-semigroups, possibly allowing a new proof for a result of Herzog on complete intersections. In the following note it is shown that this is the case.

We use the following facts about Gorenstein rings: If $R$ is a onedimensional noetherian local ring with maximal ideal $m$ and full ring of quotients $Q(R)$, then the following conditions are equivalent:

(a) $R$ is a Gorenstein ring (by definition: $m$ contains a nonzero divisor, which generates an irreducible ideal).

(b) Each principal ideal, generated by a nonzero divisor, is irreducible.

(c) The length of the $R$-module $m^{-1} / R$ is 1 .

(d) For each ideal $a$ of $R$, which contains a nonzero divisor, $\left(a^{-1}\right)^{-1}=a$. Here the inverse of an ideal is taken in $Q(R)$. For easy proofs of these equivalences see Berger [3].

Let $\bar{R}$ be the integral closure of $R$ in $Q(R)$ and $f$ the conductor from $R$ to $\bar{R}$. If $\bar{R}$ is a finitely generated $R$-module, then $f$ contains a nonzero divisor. If $R$ is Gorenstein, then the length of the $R$-module $\bar{R} / f$ is $2 d$, where $d$ is the length of $R / f$. Roquette [5] gives the following simple proof: If

$$
f=a_{0} \subset \cdots \subset a_{d-1} \subset R
$$

is a maximal chain of ideals in $R$, then

$$
f=a_{0} \subset \cdots \subset a_{d-1} \subset R \subset a_{d-1}^{-1} \subset \cdots \subset a_{0}^{-1}=f^{-1}=R
$$

is a maximal chain of $R$-submodules of $\bar{R}$, because of $\left(a^{-1}\right)^{-1}=a$, hence length $(\bar{R} / f)=2 d$.

A local complete intersection is always a Gorenstein ring and by a theorem of Serre [6] each Gorenstein ring $R$, which has embedding dimension $\leqq \operatorname{dim} R+2$, is a complete intersection.

Now assume that $R$ is analytically irreducible. This condition is equivalent with: $\bar{R}$ is a finitely generated $R$-module and a discrete valuation ring. Let $v: Q(R) \rightarrow Z$ be the corresponding valuation. We

Received by the editors March 19, 1969. 
want to give a necessary and sufficient condition for $R$ to be Gorenstein in terms of the value-semigroup $v(R)$ of $R$.

Definition. A semigroup $S$ of natural numbers (with $0 \in S$ ) is called symmetric, ${ }^{1}$ if there is an integer $n$, such that the mapping $Z \rightarrow Z$, given by $z \rightarrow n-z$, maps elements of $S$ onto nonelements and nonelements onto elements of $S$.

If $S$ is a semigroup with 0 , generated by natural numbers $n_{1}, \cdots$, $n_{t}$ with $\left(n_{1}, \cdots, n_{t}\right)=1$, then there is an integer $n \notin S$ such that $n+i \in S$ for $i=1,2, \cdots$. For each $s \in S$ we have $n-s \notin S$. So the number of elements of $S$ in $\{0,1, \cdots, n\}$ is always less than or equal to the number of nonelements.

LEMma. $S$ is symmetric iff in the set $\{0,1, \cdots, n\}$ there are as many elements of $S$ as nonelements.

Proof. If the condition is satisfied, then for $z \in\{0,1, \cdots, n\}$, $z \notin S$ we must have $n-z \in S$, hence $S$ is symmetric. Conversely, if $S$ is symmetric, then the element $n$ in the lemma equals the $n$ in the definition of a symmetric semigroup. Since $z \rightarrow n-z$ maps $\{0,1, \cdots, n\}$ onto itself, there are in this set as many elements of $S$ as nonelements.

THEOREM. Let $R$ be a one-dimensional analytically irreducible noetherian local ring, $\bar{R}$ its integral closure in the quotient field $K$ and $v: K \rightarrow Z$ the corresponding valuation. Assume $R$ and $\bar{R}$ huve the same residue class field (which is f.i. the case, if the residue class field of $R$ is algebraically closed). Then $R$ is Gorenstein iff the value-semigroup $v(R)$ is symmetric.

Proof. Choose $n$ for $v(R)$ as above and let $c=n+1$. We claim: The conductor $f$ from $R$ to $\bar{R}$ is the set of all $x \in \bar{R}$ with $v(x) \geqq c$. Obviously $f$ must be contained in this set. On the other hand, if $x \in \bar{R}, v(x) \geqq c$, then $v(x)=v(r)$ for some $r \in R$. Since $R$ and $\bar{R}$ have the same residue class field, there is a unit $e$ of $R$ such that $v(x-e \cdot r)>v(x)$. By induction there is also an $r^{\prime} \in R$ with $v\left(x-r^{\prime}\right) \geqq c^{\prime}$, where $c^{\prime}$ is the least value of an element of $f$. All elements $y \in \bar{R}$ with $v(y) \geqq c^{\prime}$ are in $R$, so $x \in R$. Now it is also clear that $x \in f$.

Assume that $v(R)$ is symmetric and $x \in m^{-1}, x \in R$. If $v(x) \in v(R)$, we can find by a similar argument as above an $r \in R$ such that $v(x-r)$ $\notin v(R)$. We still have $x-r \in m^{-1}$ and we may assume therefore that $v(x) \notin v(R)$. If $v(x)<n$, then, since $v(R)$ is symmetric, $n-v(x) \in v(R)$.

${ }^{1}$ Herzog [4] calls these semigroups "Sylvester-semigroups." For more details see his paper and also [1] and [2]. 
Choose $r_{1} \in R$ with $v\left(r_{1}\right)=n-v(x)$. Then $v\left(r_{1} x\right)=n$ and hence $r_{1} x \notin R$, contradicting $x \in m^{-1}$. So $v(x)=n$ and $m^{-1}$ contains besides $R$ only elements of value $n$. This implies that $m^{-1} / R$ is an $R$-module of length 1 and hence $R$ is Gorenstein.

Assume now that $R$ is Gorenstein and that $v_{0}<v_{1}<\cdots<v_{d-1}$ are those numbers in $\{0,1, \cdots, n\}$, which are values of elements of $R$. Define $a_{i}$ as the set of all elements $r \in R$ with $v(r) \geqq v_{i}$ $(i=0, \cdots, d-1)$. Then

$$
R=a_{0} \supset a_{1} \supset \cdots \supset a_{d-1} \supset f
$$

is a strictly decreasing sequence of ideals of $R$. Moreover this sequence is maximal, because, if we adjoin to $a_{i}$ an element $r \in R$ of value $v_{i-1}$, then we get all of $a_{i-1}$. It follows that $d=$ length $(R / f)$. Since $R$ is Gorenstein, we have $c=n+1=2 d$ and by the lemma $v(R)$ is symmetric.

COROllary (ApÉry [1]). Let $R$ be an analytically irreducible noetherian local ring of dimension 1 , whose residue class field is algebraically closed and whose maximal ideal is generated by 2 elements. Then the value-semigroup of $R$ is symmetric.

In fact, $R$ is a Gorenstein ring. For an easy direct proof see [3].

COROLlary 2. Let $R$ be an analytically irreducible noetherian local ring of dimension 1 , whose residue class field is algebraically closed and whose maximal ideal is generated by 3 elements. Then $R$ is a complete intersection iff its value-semigroup is symmetric.

By the theorem of Serre, mentioned above, Gorenstein ring and complete intersection here mean the same.

Corollary 2 gives a new proof for the local part of a result of Herzog [4], which states that an affine space-curve $C$ with the parametric equations

$$
x=t^{a}, \quad y=t^{b}, \quad z=t^{c} \quad(a, b, c \text { natural numbers with }(a, b, c)=1)
$$

is idealtheoretically a complete intersection (globally), iff the semigroup $S$, generated by $a, b, c$ is symmetric. In fact, $S$ is the valuesemigroup of the local ring $R$ of $C$ at the origin and $R$ is analytically irreducible.

\section{REFERENCES}

1. R. Apéry, Sur les branches superlinéaires des courbes algébriques, C. R. Acad. Sci. Paris 222 (1946), 1198-1200. MR 8, 221.

2. A. Azevedo, The Jacobian ideal of a plane algebroid curve, Thesis, Purdue University, Fort Wayne, Ind., 1967. 
3. R. Berger, Über eine Klasse unvergabelter lokaler Ringe, Math. Ann. 146 (1962), 98-102. MR $27 \# 175$.

4. J. Herzog, Generators and relations of Abelian semigroups and semigrouprings Thesis, Louisiana State University, New Orleans, La., 1969; Manuscripta Math. (to appear).

5. P. Roquette, Über den Singularitätsgrad von Teilringen in Funktionenkörpern, Math. Z. 77 (1961), 228-240. MR 24 \#A730.

6. J. P. Serre, Sur les modules projectifs, Séminaire Dubreil-Pisot 1960/61, Exposé 2, fasc: 1, Secrétariat mathématique, Paris, 1963. MR 28 \#3911.

Louisiana State University, Baton Rouge, Louisiana 70803

University of Heidelberg, Heidelberg, Federal Republic of Germany 